\title{
BIBECHANA
}

ISSN 2091-0762 (Print), 2382-5340 (Online)

Journal homepage: http://nepjol.info/index.php/BIBECHANA

Publisher: Department of Physics, Mahendra Morang A.M. Campus, TU, Biratnagar, Nepal

\section{Study of two-photon absorption and optical limiting}

\author{
Bal Ram Ghimire \\ Central Department of Physics, Tribhuvan University, Kirtipur \\ Email: balram.ghimire@cdp.tu.edu.np
}

\section{Article Information:}

Received: October 20, 2020

Accepted: February 9, 2021

Keywords:

Photon

Absorption

Optical

Limiters

Irradiance

Semiconductors

\begin{abstract}
Simultaneous absorption of two photons of identical or different frequencies to excite a system that is atom or molecule from ground state to excited state has great importance. It has important role as a spectroscopic tool for determining the positions of energy levels that are not connected to the atomic ground state by the one photon transition. In this work experimental study of two photon absorption of fused silica, quartz crystal and metal halides and optical limiters based on the semiconductors are studied. It was investigated that two photons absorption depletes the transmitted beam but carrier defocusing spreads the beam in space and hence density is reduced. It was realized that limiters with thin sample have low dynamic range and fluency is high on the damage prone surface and irreversible damage occurs. Some Semiconductors with high nonlinearity are found to be useful materials for optical limiters to protect devices like sensors.
\end{abstract}

DOI: https://doi.org/10.3126/bibechana.v18i2.34838

This work is licensed under the Creative Commons CC BY-NC License. https://creativecommons.org/licenses/by-nc/4.0/

\section{Introduction}

The process of simultaneous absorption of two photons of identical or different frequencies to excite a system that is atom or molecule from ground state to excited state is called two-photon absorption. In other words, in the process of two photon absorption (TPA) the transition between two states of the atom takes place by simultaneous absorption of two photon. This process was first reported experimentally by Kaiser and Garrett in 1961 [1]. Two photon absorption is useful to spectroscopic tools for determining the positions of energy levels that are not connected to the atomic ground state by a one photon transition. The absorption cross section describing this process increases linearly with laser intensity according to the relation [1].

$$
\sigma=\sigma^{(2)} I
$$

where $\sigma^{(2)}$ is a coefficient that describes twophoton absorption. The atomic transition rate due to two photon absorption scales as the square of the laser intensity [1],

$$
\mathrm{R}=\frac{\sigma^{(2)} I^{2}}{\hbar \omega}
$$

Devices designed to have high transmittance for low level inputs while blocking the transmittance for high intensity laser beams, are optical limiters. The 
first optical limiters for $\mathrm{cw}$ laser were based on the thermal lensing in absorbing liquid that is heating reduced the index causing thermal blooming resulting in a beam that was no longer focused in an imaging system. It is desirable for a limiter to keep its transmitted focusable energy below about $1 \mathrm{~mJ}$ even for inputs up to many milli Joules, while maintaining a linear transmittance of approximately $50 \%$. Two photon absorption, self-focusing in Kerr liquids, nonlinear scattering from carbon particle suspensions and among other processes have been suggested for pulsed laser sources. The focusable energy depends on the particular type of system the limiter is to protect. For example, for the human eye, a good measure of the focusable energy is that which falls within a $1.5 \mathrm{mrad}$ diameter in the final focal plane. The device must have a high damage threshold. Basic requirement for a material to be good optical limiter are: (1) High damage threshold, (2) Fast response, (3) An excited state absorption cross-section that exceeds the good state absorption cross-section. To meet all these demanding specifications research has progressed on two fronts. The first is to synthesize and characterize new limiting materials and second is to design new optical geometries that maximize the range of protection using available materials. For a limiter it must have a high linear transmission for low input energy or power and large dynamic range at which device damage to the limiting input. The ideal optical limiter has the characteristics as shown in the figure-1. Main application of the optical limiter is for sensor protection and damage to detectors is almost always determined by fluence that is irradiance, these are the quantity of interest for the output of the limiter.

For high transmission at low input, we must have low linear absorption. These criteria lead to the use of two-photon absorption process is shown in fig-2.

In 1964-1967 Leite et al. described nonlinear refraction as a means to measure low absorption in liquids and demonstrated an optical limiter that used thermal blooming in Nitrobenzene and along with a spatial filter, regulated the output power of a cw Argon laser to 30mW. In 1966 Reickhoff reported irradiance dependent self-defocusing in liquids. In 1969 Geusic et al. reported limiting behavior in $\mathrm{Si}$ attributed to stepwise nonlinear absorption with 1.06 micrometer radiation. Bogges et al. shoed fluence limiting in $\mathrm{Si}$ that was due to combination of nonlinear absorption with a refractive contribution induced by the photoexcitation of free carriers. Ralston and Chang described the use of two photon absorption for optical limiting and conducted power limiting experiments in a series of semiconductors like GaAs, CdSe, and CdS. In $1980 \mathrm{CS}_{2}$ was tested as a limiting medium. An apparent advantage of liquid-based limiters is that they self-heal, permitting high dynamic ranges limited only by damage to cell windows. The response time has been shown to be $2 \mathrm{p}$ sec in the visible. The limiting power can be varied by adjusting the concentration of $\mathrm{CS}_{2}$ in solvents. Soileau et al. described that when picoseconds pulses were used in a comparative study of limiting in seven liquid crystals, two photon absorption was responsible for the limiting behavior. Bjorkholm et al. built advice that uses the selffocusing in sodium vapor that is due to near-resonant excitation at a wavelength of $590 \mathrm{~nm}$. Some of the largest nonlinearities exhibited to date are in semiconductors.

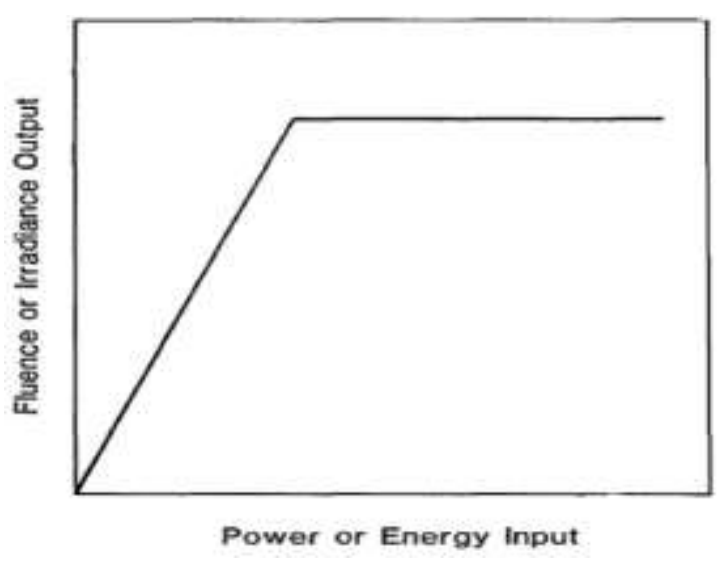

Fig. 1: Irradiance output of an ideal Optical Limiter as a function of input power of energy [2]. 
In this work description of the experimental determination of two-photon absorption coefficient in fused Silica, crystalline quartz and some alkali halides are given on the basis of Stephen A. Slattery and co-workers. Report of the detailed operational characteristics and a theoretical description of optical limiters based on two-photon absorption and the subsequent photo-generated free-carrier defocusing in semiconductors are given.

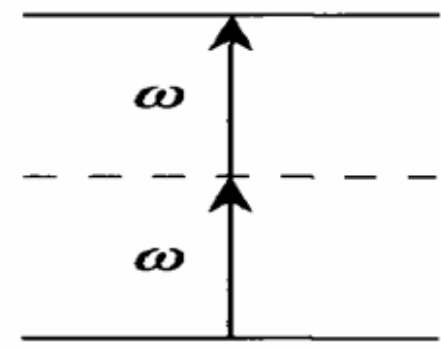

Fig. 2: Two photon absorption.

\section{Theory and Methodology}

Experimental determination of two-photon absorption coefficient

In 2020 Nugroho et al. realized that for many
The energy stability of $211 \mathrm{~nm}$ radiation was $100 \mu \mathrm{J}$ [ 5, 6,7,8, 9]. To achieve the time coincidence between the interacting pulses inside the nonlinear crystal the manually optical delay was used. To control the energy incident on the energy detector small part of UV pulse was reflected to the energy detector by a selected Suprasil fused Silica plate at near normal incidence. There is a plane-convex $\mathrm{CaF}_{2}$ lens to change the cross-sectional area of the laser beam. The samples were placed on a mountable table along the axis of UV beam behind the lens. There is another detector placed $10 \mathrm{~cm}$ in front of the focal point which restrict the movement of sample towards the beam focus in order to protect it from damage [5].

Similarly, Hagen et al. calculated two photon absorption coefficients of ten different semiconductors using the following model [11].

$\beta=k \sqrt{E_{p}} \frac{F_{2}(2 \hbar \omega) / E_{g}}{n^{2} E_{g}^{3}}$

where $\mathrm{n}$ is linear refractive index $\mathrm{E}_{\mathrm{p}}$ and $\mathrm{E}_{\mathrm{g}}$ are in electron volts, constant $\mathrm{k}$ is was found to be 2600 to 3600 .

applications potential can be hold by two photon absorption and predicted that two-photon absorption occur coherent and incoherent biexcitation transition [3]. Stephen A. Slattery and D. N. Nikogasyan described two photon absorption properties of some common UV materials like fused silica, quartz, $\mathrm{CaF}_{2}$, and $\mathrm{BaF}_{2}$, using the fifth harmonic of Nd-glass laser radiation at $211 \mathrm{~nm}$. They determined TPA coefficient with the help of following experimental set up as shown in the fig-3. The pulses at fundamental frequency at $1055 \mathrm{~nm}, 800$ $\mu J$, with repetition rate $27 \mathrm{~Hz}$ and fourth harmonic at $264 \mathrm{~nm}, 400 \mu \mathrm{J}$, with repetition rate $27 \mathrm{~Hz}$ were directed into the $0.2 \mathrm{~mm}$ thick beta-

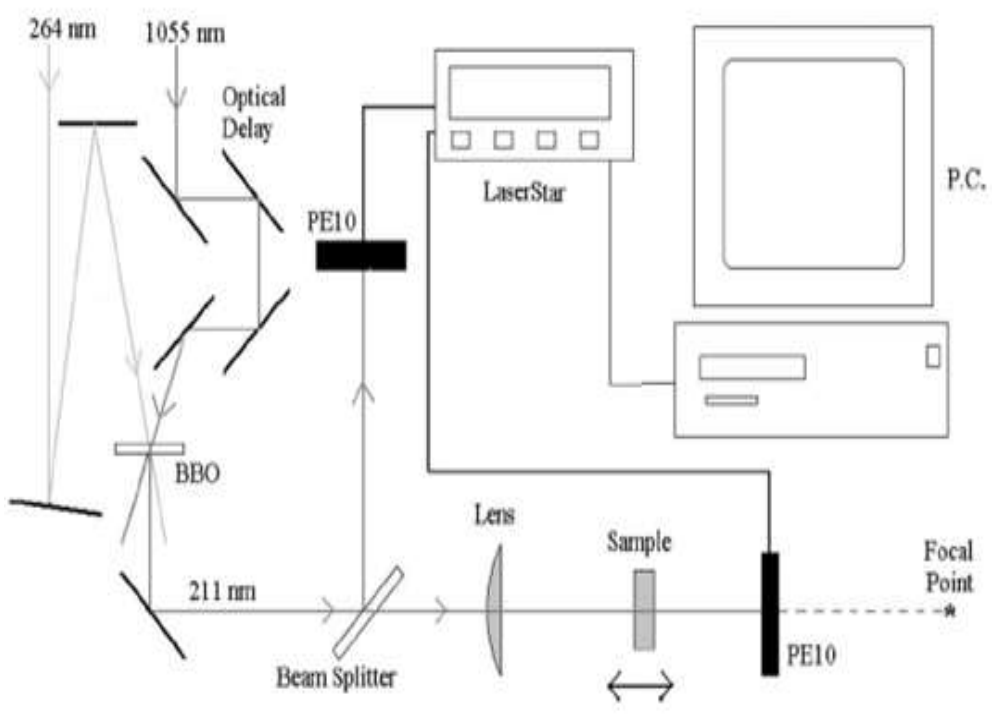
Barium-Borate ( $\beta-\mathrm{BaB}_{2} \mathrm{O}_{4}$ ) crystal cut for first type nonlinear Sum-frequency Mixing such that the angle between two pulses was about $7^{0}$ in vertical plane.

Fig. 3: Experimental set up for determination of two photon absorption coefficient [3]. 


\section{Optical limiting with semiconductors}

E. W. Van Stryland and coworkers described the optical limiting with semiconductors. In 2020 Qian et al. demonstrated reflection-mode pulse limiters using nano-scale refractory films of Aluminium based metallic quantum well and investigated that such a device could provide large and ultra-fast Kerr-type optical non-linarites [4].

When the thick samples of semiconductor like $\mathrm{ZnSe}$, GaAs were used, the large nonlinearities of semiconductor could be used to prevent damage [5, $6,10,11]$. When the light was focused tightly into the bulk of the materials, the nonlinear absorption combines with nonlinear refraction and keeps the irradiance within the semiconductor below the damage threshold and becomes self-protecting. If low input limiting threshold is maintained the irradiance reduces on the surface, at high inputs the two photon absorption and defocusing reduce the irradiance in the bulk preventing damage. Such situation cannot be explained quantitatively, though it can be explained qualitatively [2].

Schematic drawing of the thick limiter geometry has been shown in the figure 5. The solid lines show linear beam propagation for low inputs and the dashed lines show the beam for high inputs. Two photons absorption depletes the transmitted beam but carrier defocusing spreads the beam in space and hence density is reduced. It was noted that limiters with thin sample have low dynamic range and fluence is high on the damage prone surface and irreversible damage occurs within 1 or 2 orders of magnitude of limiting. To reduce this problem is to use thick samples having large thickness than depth of the focus and to focus into the bulk of the materials reducing the irradiance on the damage prone -surface. At low inputs the thick limiter acts linearly as thin limiter. But at thin limiter's threshold thick limiter does not act as linear at the region within the focal volume. Because at that region the irradiance become high enough to have significant two photon absorption along with carrier defocusing.

\section{Monolithic optical limiter}

Optical limiters for the visible based on $\mathrm{ZnSe}$ and $\mathrm{ZnS}$ were built by the process of combination of absorption and refraction. These limiters work well for picoseconds inputs [11]. The geometrical arrangement of a monolithic optical limiter has been given in the figure 7 . The solid lines show the optical path for inputs and dashed lines represent the optical paths for high inputs. This device acts as a unity power inverting lens for the low inputs. The design of the monolithic optical limiter takes the damageprone surface as far from focus as is possible to maintain high irradiance within the bulk, so that there would be maximum dynamic range. The out of the mode locked Nd: YAG laser was collimated to a spot size of $1 \mathrm{~mm}$ and directed into the device. For ZnSe device limiting energy was found to be 10nJ with $300 \mathrm{~W}, 30 \mathrm{ps}$ and FWHM into $0.53 \mu \mathrm{m}$ pulse and dynamic range $10^{4}$.

The optical damage to the bulk of the material was prevented by combining the effect of depletion due to TPA and carrier defocusing before the focal position determined by linear optics. Thus monolithic limiter is self-protecting against high irradiance nano and picoseconds pulses. When they focused nanoseconds pulses less tightly into plane parallel to $\mathrm{ZnSe}$ and $\mathrm{ZnS}$ samples, they found that the devices suffered bulk damage. But when they focused tightly the focus volume become small and the thermal nonlinearity. caused damage. However, problem can be overcome by using materials with a negative thermal nonlinearity.

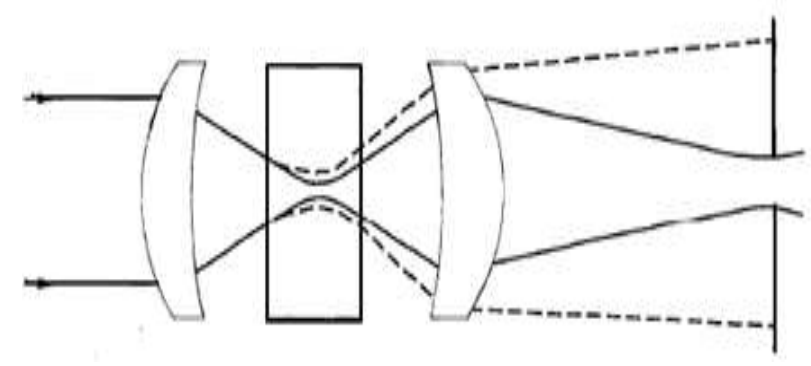

Fig. 4: Schematic diagram of thick limiter geometry. 


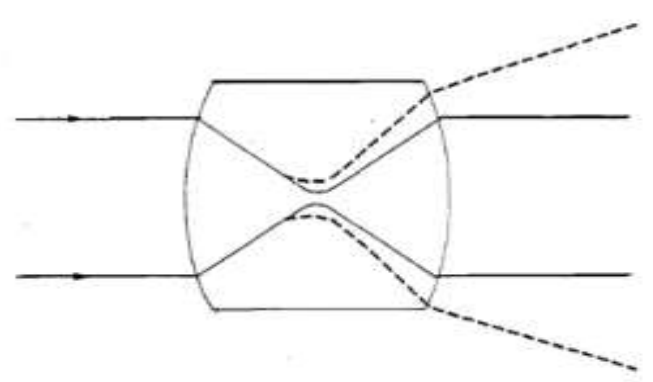

Fig. 5: Monolithic Optical limiter [2].

\section{Result and Discussion}

The UV beam irradiance was varied in the range $2-40 \mathrm{GW} / \mathrm{cm}^{2}$ At room temperature incident and transmitted pulse energies were measured by using Photo Electric detector PE10 connected to Laser Star energy meter system. Here the position of the sample is so adjusted that allowed them to avoid selffocusing. When these conditions were fulfilled there would be no break-up and filamentation of beam. They recorded the transmittance irradiance dependencies and fitted them with the theoretical curve using Mathematica package that has been shown in fig- 4 . The beam diameter was measured by measuring the energy transmittance through an aperture placed at the center of the beam symmetrically around the optical axis. The average value for the beam diameter at FWHM was $2.2 \pm 0.1$ $\mathrm{mm}$ and pulse duration value at $211 \mathrm{~nm}$ was $250 \pm$ $20 \mathrm{fs}$ and $220 \pm 10 \mathrm{fs}$ at 264 at 1/e level.

By measuring the beam diameter and pulse duration they calculated the values of two photon absorption coefficients of samples at different wavelengths which are given in the table-1.

Van Stryland and co-workers observed that at the aperture $30 \mathrm{~cm}$ behind the second lens, of the thick ZnSe optical limiter the transmitted energy varies with incident energy as shown in the figure 6 . Where (a) represent the linear transmission, (b) represents the effect of two photon absorption showing all energy collected and (c) indicates the transmitted energy through an aperture as a function of the input $0.53 \mu \mathrm{m}$ picoseconds pulses.

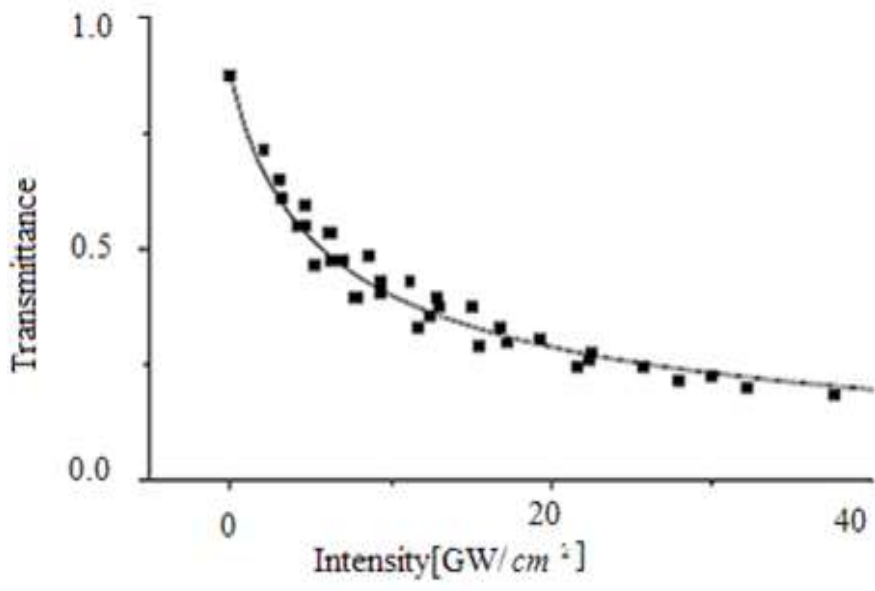

Fig. 6: Transmittance as a function of incident LASER intensity for fused Silica [2].

Table-1: Two photon absorption coefficients of samples at different wavelengths.

\begin{tabular}{|l|l|l|}
\hline Materials & $\lambda(\mathrm{nm})$ & $\beta\left(10^{-11} \mathrm{~cm} / \mathrm{W}\right)$ \\
\hline Fused Silica & 211 & $50 \pm 8$ \\
& 248 & $4.5 \pm 2.9$ \\
& 264 & $1.7 \pm 0.2$ \\
Crystalline & 211 & $59 \pm 8$ \\
Quartz & 264 & $1.2 \pm 0.2$ \\
& 211 & $58 \pm 9$ \\
Barium Fluoride & 258 & $11 \pm 7$ \\
& 211 & $26 \pm 5$ \\
Calcium Fluoride & 248 & $0.8 \pm 0.5$ \\
\hline
\end{tabular}

This shows that the limiting is weakly dependent on the focal length of the second lens and distance to the aperture. With the help of limiter at this configuration using picoseconds $0.5 \mu \mathrm{m}$ pulses and tight focusing they obtained limiting energies down to $14 \mathrm{~nJ}$ corresponding to a peak power of $400 \mathrm{w}$ with dynamic range 1000 . 


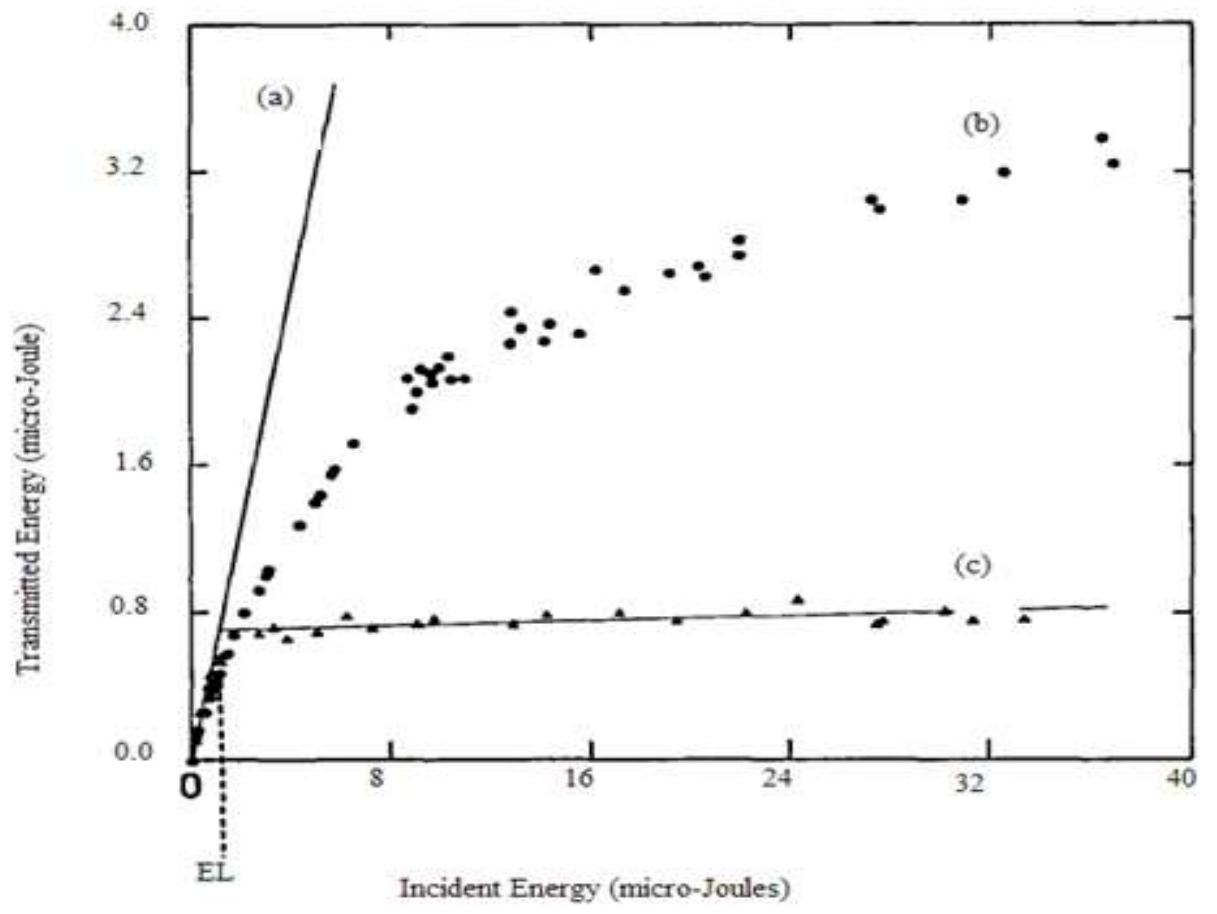

Fig. 7: Transmitted energy as a function of incident energy [2]

\section{Conclusion}

Process of two-photon absorption and experimental determination of two photon absorption coefficients of fused silica, quartz crystal and metal halides are discussed, on the basis of model given by Stephen A. Slattery and coworkers. Working of optical limiters based on the semiconductors with the help of the model given by Van Stryland and co-workers was studied. It is investigated that two photons absorption depletes the transmitted beam but carrier defocusing spreads the beam in space and hence density is reduced. It was noted that limiters with thin sample have low dynamic range and fluency is high on the damage prone surface and irreversible damage occurs. Optical limiters based on the process of two photon absorption are better and cheaper devices to protect the expensive and sensitive devices like sensor.

\section{References}

[1] R. W. Boyd, Academic Press, 2003, Pp. 528-530.
[2] S. Eric, Y. Y. Wu, D. J. Hagan, M. J. Soileau, and K. Mansour J. Opt. Soc. B 5 (9) (1988) 1228-1235.

[3] B. S. Nugroho, A. A. Iskandar, V. A. Mallyshev and J. Knoester, Phy Rev. B 102 (2020) 045405

[4] H. Qian, S. Li, Y. Li, C. F. Chen, W. Chen, S. E. Bopp, Y. U. Lee, W. Xiong and Z. Liu, Science Advances 6 (2020) 3456 . https://doi.org/10.1126/sciadv.aay3456.

[5] S. A. Slattery and D. Nikogosyan, Optics Communications 228 (2003) 127-131.

[6] J. M. Hales, D. Hagan, and S. Eric, Journal of Chemical Physics 121 (2004) 1101-1106.

[7] M. Balu, J. Hales, D. Hagan, and S. Eric, Optical Society of America 9 12(2004) 3820.

[8] R. A. Negres, J. Hales J, A. Kobyakob, D. Hagan, and S. Eric, Optics Letters 27 (2002) 1980-1088

[9] I. K. Dmitriy, S. Yang, D. Hagan, and S. Eric Applied Optics 38 (1999) 5168-5180

[10] K. Delfield, L. Yong, R. Negres, F. Meigong,and H. Florencio, Chem. Mater. 14 (2002) 3663-3667.

[11]D. J. Hagan, E. W. Van Stryland, M. J. Soileau, and Y. Y. Wu, J. Opt. Soc. Am A 3(13) (1986) 105. 